\title{
EXACT CURVATURE ELASTICA OF A THIN CANTILEVER UNDER TERMINAL LOADS
}

\author{
GIOVANNI MINGARI SCARPELLO AND DANIELE RITELLI
}

\begin{abstract}
Communicated by Mauro Spera
Abstract. A thin, flexible, one side-built-in rod under a concentrated terminal force is studied in its elastic equilibrium configuration. In order to make the problem more tractable, a proper set of state variables is chosen, facing with a second order, nonlinear, but autonomous boundary value problem, in the rotation $\varphi(s)$ pertaining to each $s$-section. The search of the free end rotation $\varphi_{0}$, following the isoperimetric assumption, leads to a numerical sub-problem inside the main BVP. Furthermore, if $x(s)$ and $y(s)$ mean the elastica coordinates parametrized on the arclength $s$, one obtains $x^{\prime}(s)$ and $y^{\prime}(s)$ as elliptic functions of $s$. Finally, some minor changes have been shown in order to pass from a loading force to a more general free-end load combination, consisting of a force and a couple.
\end{abstract}

\section{Introduction}

The bending of an elastic beam under various loads provided the first problem of practical importance to theory of elasticity, and fed the initial development of beam theory, due to the names of Jakob and Johann Bernoulli, Euler and Lagrange. One of the simplest ones to consider is the loaded beam rigidly supported at one end only: a simple cantilever, whose exact shape is required when inflected under terminal loads. Exact means: taking into account its exact curvature. Then we wish to describe mathematically the shape assumed by a flexible rod builtin at one end, and affected at its free end by a general co-planar load consisting of a slender force and a bending couple. Such a cantilever is commonly treated assuming an approximate curvature expression and obtaining a third order deflection curve; but our viewpoint is different, and aims at giving an exact solution to the problem, assuming the exact curvature. As we told, such a problem is a very old one: its birth preceded the existence of calculus tools capable of solving it. 


\section{A Recent Literature's Background}

A historical problem's outline can be read in [9]. Here it will be enough to recall that a completely different model dates back to G. Kirchhoff, Über das Gleichgewicht und die Bewegung eines unendlich dünnen elastischen Stabes, 1859. He had demonstrated [8] that under forces applied only to ends of a straight-linear rod, that the same equilibrium equations can also describe the dynamics of a rigid body around a fixed point (spinning top). Two recent papers stress the methods of Kirchhoff analogy achieving a fairly complete classification of ideal elasticas through a detailed comparison with the spinning top motions. In the first it is shown [11] how to classify the shapes of Kirchhoff filaments based on the geometry of the spinning top solutions, pushing the Kirchhoff analogy to its extreme, and obtaining properties of filaments. In the second [10], the configurations of twisted elastic rods under applied end loads and clamped condition are investigated and classified as authors claim all possible equilibrium states of straight rods by labelling them according to the number of critical points of the curvature along the centre line. The tables put at the end, anyway do not seem to be completely exhaustive as declared. Finally we came to know of three further articles, [3], [5] and [6], to be included in our literary outline on the large deflections of a cantilever, and of these [5] and [6] are issued in the same Journal. The oldest [3], deals shortly with a clamped rod under a force parallel to the wall where the rod is built in, and perpendicular to it: the elastica's shape is not computed, but the free end large deflection is evaluated through elliptic integrals of I and II kind. It is then the less studied case because its loading condition, avoiding any complication, is also of a scarce utility. The second [5], treats the subject more generally, aiming to a mathematical model for the flexible arms of compliant mechanisms subjected to any loading. After the cantilever elastic model has been introduced, the authors obtain the final horizontal and vertical position of the rod's free end. Their idea is that a "pseudorigid body model" can be accordingly developed and used for providing parametric evaluations which best approximate the beam deflection and stiffness. The most recent paper [6], covers with greater details all the topics of the previous one. The authors, following a path different from ours, arrive at formulae similar to our $x(\varphi)$ and $y(\varphi)$ in Section 4, but the resemblance stops here. In fact we chose to work with the arclength $s$ as a state independent variable, and tackling integrals consisting of rational combinations of Jacobian elliptic functions, see formulæ(18) and (19). In such a way we obtain elastica parametric equations, see formulæ (21) through $s$ as: a linear term plus an elliptic integral of III kind plus "sd" elliptic function. On the contrary, Kimball and Tsai 
go on with the angular deflection $\theta$, and tackle the integration of

$$
\frac{\mathrm{d} \theta}{\sqrt{a+b \sin \theta+c \cos \theta}}, \quad \frac{\cos \theta \mathrm{d} \theta}{\sqrt{a+b \sin \theta+c \cos \theta}}, \quad \frac{\sin \theta \mathrm{d} \theta}{\sqrt{a+b \sin \theta+c \cos \theta}}
$$

developing a long chain of transformations for their reduction to Legendre I and II kind normal forms. In such a way they compute the final coordinates of the free end (vertical and horizontal deflection), making a deep analysis in order to distinguish when the line has an inflection point or not. All the pseudorigid body modeling is improved, and a final optimization is added to find the model parameters which best approximate the deflections of the actual beam. Neither of the articles, [3], [5] and [6] is concerned with the global solution to the two points problem, or with the range of rodlenghts, e.g. like our (8), for which the solution's uniqueness is ensured. Then all the aspects for which uniqueness of the solution is ensured to the Dirichlet boundary value problem were there completely ignored.

\section{Paper Organization}

As far as it concerns the strain, we will use the exact curvature formulation, as done in our previous paper [9], devoted to the simply supported beam with flexure induced by the axial compression. Let $\Omega x y$ be a reference system with origin $\Omega$ at rod's free end, the $\Omega x$ axis, superimposed to the unstrained rod, is oriented towards the clamping, and $\Omega y$ downwards. Let $y=y(x)$ be the unknown cartesian equation of the planar elastica. If the rod's free end is loaded by a slender force, its axial component does induce at each transverse $S$ - section a flexure of moment $M_{a}$ whose arm is depending on how $S$ is deflected. On the contrary, the bending couple $M_{v}$ due to the vertical load component, will depend on its $x$-distance from $S$. The bending moment at $S$ is therefore the addition of a endogenous one, say $M_{a}(y)$ dependent on size $y$ of the strain itself, plus an endogenous one, say $M_{v}(x)$. The latter is responsible for the loss of autonomy of the ODE to $y=y(x)$, and then of its non-integrability. In other words, the traditional relationship providing the elastica's curvature $c\left(y^{\prime}, y^{\prime \prime}\right)$ as a ratio of the bending moment to the flexural rigidity $E J$

$$
c\left(y^{\prime}(x), y^{\prime \prime}(x)\right)= \pm \frac{M}{E J}= \pm \frac{1}{E J}\left[M_{a}(y)+M_{v}(x)\right]
$$

do not fit to our specific problem. Therefore we will go back to the vectorial formulation, EVES, of course to be properly specialized. We will get a two point boundary value problem whose unknown is the rotation $\varphi(s)$ of a generic rod cross section $S$ as a function of its distance $s$ along the strained centerline. The 
thin rod's free end will rotate of an amount $\varphi_{0}$ whose computing is compulsory in order to carry out the integration. Such a $\varphi_{0}$ computation allows a sub-numerical problem to be solved inside the boundary value one. In such a way $x^{\prime}(s)$ and $y^{\prime}(s)$ will come as functions of the curvilinear abscissa $s$ and the integration is performed through Jacobian elliptic functions. Such a planar problem is not treated adequately in the relevant literature which describes, see [7], exact solutions to the flexible cantilever under load parallel or normal to the unstrained centerline, but never slender, as we do.

\section{A 3D Rod Model}

The main difficulties about elastica are: the curvature intrinsic nonlinearity under non small displacements; geometric complexity of the unstressed configuration; constraint effects such as isometry (non stretching); and finally boundary conditions. The peculiarity of a thin rod under terminal forces and/or terminal couples, is that it buckles rather than breaks: our experience tells us that a compressed rod can buckle in any lateral direction. This is the strongest motivation for a spatial modelling of $i$. The curvilinear abscissa along the 3D deflection curve $\Gamma$, is $s \geq 0$ with $s=0$ at $\Omega$, and $s>0$ at $P(s)$.

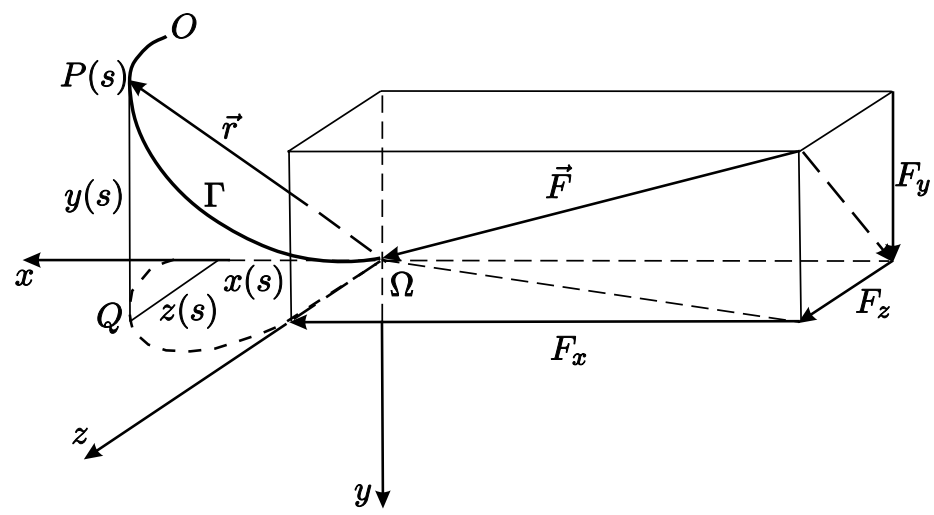

Figure 1. Sketch of a 3D rod under a load $\vec{F}$ : frame of reference $\Omega x y z$, elastica $O P \Omega$, each $s-$ section identified by vector $(P-\Omega):=\vec{r}$.

We lay down the following assumptions:

$\mathrm{A}_{1}$ the rod is thin, initially straight, homogeneous, with uniform cross section and uniform flexural rigidity. This last is the product $E J$, where $E$ is the Young modulus, and $J$ the cross section second moment of inertia about 
a "neutral" axis normal to the plane of bending and passing through the central line;

$\mathrm{A}_{2}$ the rod is unloaded along all its body, and loaded by a force $\vec{F}=$ constant at its free end. Then all the strain is due to the loads' bending moment whilst the shearing stress effects are completely ignored;

$\mathrm{A}_{3}$ we are faced with linear elasticity, but transverse deformation is ignored. Anyway the rod is not freely extendible: there is a isoperimetric constraint, and $L$ is the value of its invariant length;

$\mathrm{A}_{4}$ due to rest of static equilibrium, no rod element undergoes acceleration.

The EVES, see [7], for the deformed rod consists of two equations. The first is:

$$
E J \frac{\mathrm{d}}{\mathrm{d} s}(P-\Omega) \times \frac{\mathrm{d}^{3}}{\mathrm{~d} s^{3}}(P-\Omega)=\vec{F} \times \frac{\mathrm{d}}{\mathrm{d} s}(P-\Omega)
$$

where $\times$ is the cross product in $\mathbb{R}^{3}$. Putting (see Figure 1) $(P-\Omega):=\vec{r}$, we have

$$
E J \frac{\mathrm{d} \vec{r}}{\mathrm{~d} s} \times \frac{\mathrm{d}^{3} \vec{r}}{\mathrm{~d} s^{3}}=\vec{F} \times \frac{\mathrm{d} \vec{r}}{\mathrm{~d} s} .
$$

The EVES second one (moment) is

$$
E J \frac{\mathrm{d} \vec{r}}{\mathrm{~d} s} \times \frac{\mathrm{d}^{2} \vec{r}}{\mathrm{~d} s^{2}}=\vec{M}
$$

where $\vec{M}$ is the external bending moment. Let us specialize both of them to our elastic system depicted at Figure 2, where a one side built-in, $L$-long, thin, straight-linear flexible rod is loaded by a force $\vec{F}$ at its free end, and all the system is planar. Recalling the $\Omega x y$ reference system feature, the increasing $s$ is from $\Omega$ to the wall, so that the tangent $t$ to the elastica at a whichever point $P(s)$, has its positive sense towards the built-in end. Finally, $\Omega z$ is orthogonal to the elastica plane. Let it be $\theta$ the angle of $t$ with the oriented axis $\Omega y$.

The picture shows the cantilever simultaneously loaded by a slender pushing force and by a bending clockwise couple. The first one $\left(F_{x}>0, F_{y}>0\right)$ induces a global strain in the rod so that its free end-initially parallel to the wall, undergoes a (positive) clockwise rotation. The same happens as a consequence of the couple $M_{0}$. Let then be $\varphi_{0}>0$ the unknown total rotation of the free end. With a straightforward calculation from (2) we find non-zero projections on $\Omega z$ only

$$
M_{z}=-E J \frac{\mathrm{d} \theta}{\mathrm{d} s}
$$

while (1) gives

$$
-E J \theta^{\prime \prime}(s)+F_{y} \sin \theta-F_{x} \cos \theta=0
$$




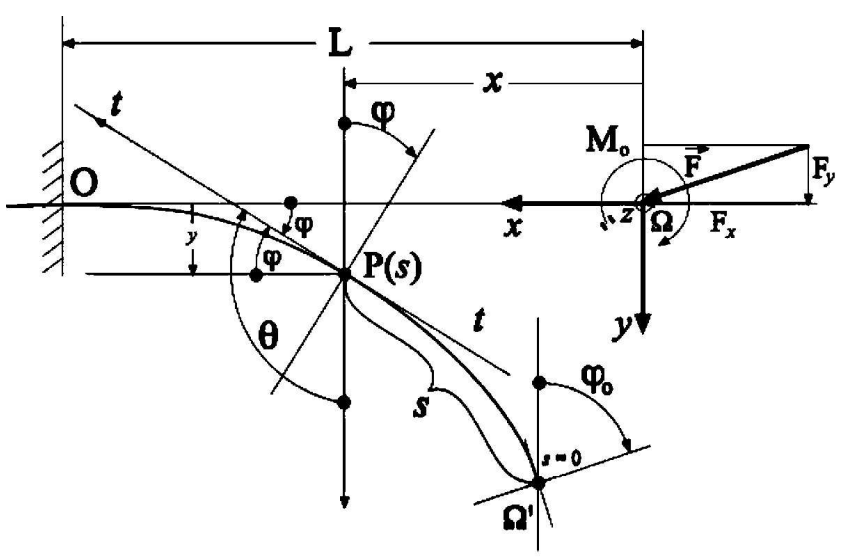

Figure 2. Sketch of a thin cantilever under a planar load: $O \Omega$ unstrained rod whose elastica is $O P \Omega^{\prime}$, each $s-$ section rotates of $\varphi$.

\section{A Planar Boundary Value Problem}

The vectorial scheme EVES generally provides six scalar equations, which in our particular case are reduced to a planar system of two scalar decoupled differential equations. Their unknown functions are: the bending moment $M_{z}(s)$ and the flexion angle $\theta(s)$ between the oriented straightline $\Omega y$ and the local $s$-tangent oriented in the sense of the growing arclengths. It is nevertheless of better convenience to introduce the rotation $\varphi$ which the $s$-section of the strained centerline undergoes with respect to its original position: $\varphi=\theta-\pi / 2$. In such a way, equation (4) becomes $-E J \varphi^{\prime \prime}(s)+F_{y} \cos \varphi+F_{x} \sin \varphi=0$. At the built-in end $s=L$ no rotation is allowed, and then $\varphi=0$. At the free end $s=0$ the bending moment is zero, and therefore also $\varphi^{\prime}(s)$ shall be zero there. So we have to solve the nonlinear two-points boundary value problem

$$
-E J \varphi^{\prime \prime}(s)+F_{y} \cos \varphi+F_{x} \sin \varphi=0, \quad \varphi^{\prime}(0)=0, \quad \varphi(L)=0
$$

Equation (5) looks like that of pendulum, but with three important differences. First, the independent variable is not time, but the arclength $s \geq 0$ of the strained centerline. Second: we are faced with a trinomial equation due to the simultaneous presence of sinus and cosine with different weights according to which $x$ or $y$ load component is prevailing. Finally, the third is that we do not have a initial value problem (IVP, Cauchy), but a boundary value problem (BVP, Dirichlet), for being the limiting conditions concerned with the ends $s=0$ and $s=L$. Such a BVP is of mixed type (II kind), because different variables are prescribed at the ends, 
namely the rotation at $s=L$ and its derivative at $s=0$. Looking to the following, (5) is better written as

$$
\varphi^{\prime \prime}(s)=\frac{1}{2}\left(\beta_{y} \cos \varphi(s)+\beta_{x} \sin \varphi(s)\right), \quad \varphi^{\prime}(0)=0, \quad \varphi(L)=0
$$

where $\beta_{x}=2 F_{x} / E J$ and $\beta_{y}=2 F_{y} / E J \in \mathbb{R}$ should not be both zero simultaneously. The equation is still nonlinear, but autonomous: in such framework we will be able to perform the explicit integration.

\section{Elastica Integration}

\section{Elastica Equations $x(\varphi), y(\varphi)$ Parametrized Via the Rotation}

As we shall see not before long, the solution $\varphi(s)$ of (6) is a strictly increasing function whose derivative $\varphi^{\prime}(s)$ is given by

$$
\varphi^{\prime}(s)=\sqrt{\beta_{y}\left(\sin \varphi-\sin \varphi_{0}\right)-\beta_{x}\left(\cos \varphi-\cos \varphi_{0}\right)} .
$$

Anyway, we shall integrate the equations $x^{\prime}(s)=\cos \varphi(s), y^{\prime}(s)=-\sin \varphi(s)$ from $s=0$ (free end rotates of $\varphi_{0}$ ) to the generic $s$-section $\varphi$, and then

$$
\mathrm{d} x=\frac{\mathrm{d} \varphi \cos \varphi}{\mathrm{d} \varphi / \mathrm{d} s}, \quad \mathrm{~d} y=-\frac{\mathrm{d} \varphi \sin \varphi}{\mathrm{d} \varphi / \mathrm{d} s}
$$

so that:

$$
\begin{aligned}
& x(\varphi)=\int_{\varphi_{0}}^{\varphi} \frac{\cos \phi \mathrm{d} \phi}{\sqrt{\beta_{y}\left(\sin \phi-\sin \varphi_{0}\right)-\beta_{x}\left(\cos \phi-\cos \varphi_{0}\right)}} \\
& y(\varphi)=-\int_{\varphi_{0}}^{\varphi} \frac{\sin \phi \mathrm{d} \phi}{\sqrt{\beta_{y}\left(\sin \phi-\sin \varphi_{0}\right)-\beta_{x}\left(\cos \phi-\cos \varphi_{0}\right)}} .
\end{aligned}
$$

The above formulæ provide closed form expressions to elastica's cartesian coordinates as functions of the $\varphi$-rotation of the rod's $S$-section. The relevant integrals could be carried out through [4], formulæ 293.06 page 180, and 293.05 page 179. Nevertheless the free end rotation $\varphi_{0}$ requires a numerical approach and the relevant value will affect heavily the integration. It is then better to detect the equation to $\varphi_{0}$, leaving to the above $x(\varphi)$ and $y(\varphi)$ expressions a numerical use for possible 
checks. In order to gain closed form expressions, first we will solve the boundary value problem (6): in such a way, by $\varphi(s)$ both $x^{\prime}(s)$ and $y^{\prime}(s)$ will come as functions of $s$ and a further integration will provide $x$ and $y$ through $s$. What above, after having localized the free end rotation $\varphi_{0}$, will allow to complete the shooting, arriving to its analytical expression via a first kind elliptic integral.

\section{The Strained Rod Arclength}

First of all we ask what can be inferred about the strained rod $\mathfrak{R}$. If, for instance, we take into account only shortening and extension effects on the rod (neglecting shear) due to $\vec{F}$, the change $\left|\Delta L_{N}\right|$ in arclength will be

$$
\left|\Delta L_{N}\right|=\frac{1}{E A} \int_{\mathfrak{R}} N(s) \mathrm{d} s .
$$

Figure 3 shows that at $(s, \varphi)$ of elastica, the total normal stress $N$ will be

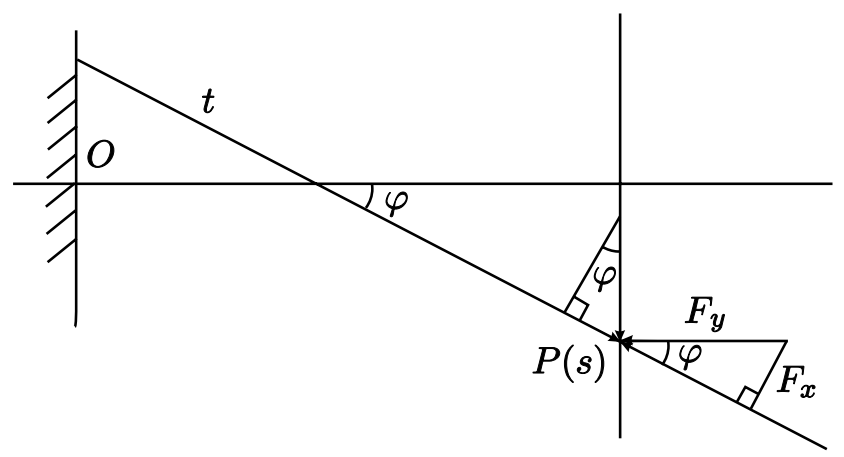

Figure 3. Geometric elements for evaluating the axial stress at $P(s, \varphi)$.

$$
N(\varphi)=F_{y} \sin \varphi-F_{x} \cos \varphi .
$$

By Figure 2 we get: $x^{\prime}(s)=\cos \varphi, x^{\prime}(s)=-\sin \varphi$, and then

$$
\Delta L_{N}=-\frac{1}{E A}\left(F_{y} y_{\Omega^{\prime}}+F_{x}\left(L_{0}-x_{\Omega^{\prime}}\right)\right) .
$$

But the coordinates $x_{\Omega^{\prime}}$ and $y_{\Omega^{\prime}}$ of the free end "mapped" position $\Omega^{\prime}$ of $\Re$, are unknown, and $\Delta L_{N}$, generally speaking, cannot be computed in easy way. Then the rod isoperimetric constraint is assumed: while the rod's shape is changing and its the free end is pushed towards the wall, the rod's arclength is kept constant throughout all the elastic flow till to equilibrium. 


\section{The Free End Rotation $\varphi_{0}$}

The problem requires to compute the free end equilibrium rotation $\varphi(0):=\varphi_{0}$ of the $(L, E, J)$ system under the load $\vec{F} \equiv\left(F_{x}, F_{y}, 0\right)$. From now on, we will assume $\beta_{x}, \beta_{y}>0$, what is not affecting the generality, the other cases being tractable likewise. On the contrary, it is mandatory that the inequality

$$
0<L<\sqrt[4]{\frac{4}{\beta_{x}^{2}+\beta_{y}^{2}}}
$$

holds, as ensuring that (6) has one and only one solution, according to [2] theorem 6.1 page 93. As a matter of fact, problem (5) does not possess one solution if whichever $\operatorname{rod}(L, E, J)$ is loaded by whichever $\vec{F}$. In fact, by the previous things we argue that the force's size cannot be willingly large, but shall admit the upper bound

$$
F<\frac{E J}{L^{2}} .
$$

In order to face the BVP (6), one shall previously solve the auxiliary shooting initial value problem

$$
\begin{aligned}
\varphi^{\prime \prime}(s) & =\frac{1}{2}\left(\beta_{y} \cos \varphi(s)+\beta_{x} \sin \varphi(s)\right) \\
\varphi(0) & =\varphi_{0} \in[-\pi, \pi], \quad \varphi^{\prime}(0)=0
\end{aligned}
$$

detecting which conditions shall be met by $\varphi_{0}$ so that the solution to (9) is also solution to (6) too. Notice that (9) is a Weierstraß equation, which, following [1], pages 287-292, is brought to the quadratures putting

$$
\begin{aligned}
& \Phi(\varphi)=\beta_{y}\left(\sin \varphi-\sin \varphi_{0}\right)-\beta_{x}\left(\cos \varphi-\cos \varphi_{0}\right) \\
& \int_{\varphi_{0}}^{\varphi} \mathrm{d} s=\operatorname{sign}\left(\beta_{y} \cos \varphi_{0}+\beta_{x} \sin \varphi_{0}\right) \int_{\varphi_{0}}^{\varphi} \frac{\mathrm{d} \phi}{\sqrt{\Phi(\phi)}}
\end{aligned}
$$

where $\Phi(\varphi) \geq 0$. Furthermore, due to initial condition rest point of the shooting problem (9), it shall be $\Phi\left(\varphi_{0}\right)=0$, and then $\varphi_{0}$ is a stationary point (maximum or minimum) for the solution to (9), solution which will either decrease or increase till to the next (simple) root of $\Phi(\varphi)$, where the monotonicity reverses. That is depending on the clear periodicity of the solutions to (9). It is therefore essential to understand how the solutions of (9) really behave for different $\varphi_{0}$ values, in order to perform the $\varphi_{0}$ choice capable of solving (6). For the purpose, the following Lemma defines the range described by the solutions to (9) when the binomial $\beta_{y} \cos \varphi_{0}+\beta_{x} \sin \varphi_{0}$ changes its sign. We will not provide the relevant proof because this is a trivial and tedious sequence of goniometric inequalities. 


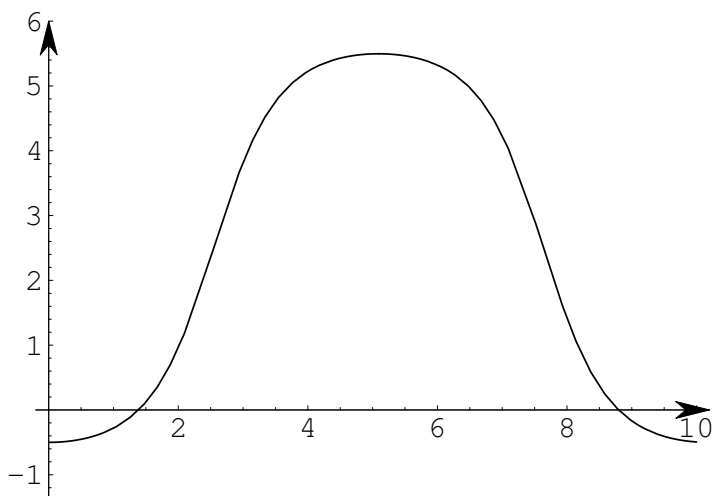

Figure 4. Solution of (9) case $\beta_{y}=3, \beta_{x}=4, \varphi_{0}=-0.5$

Lemma 1. Let it be $\varphi(s)$ the solution to (9). Then, putting

$$
r\left(\beta_{x}, \beta_{y}\right)=\frac{\beta_{x}}{\sqrt{\beta_{x}^{2}+\beta_{y}^{2}}}
$$

we have that

1. if $\beta_{x} \sin \varphi_{0}+\beta_{y} \cos \varphi_{0}>0$ then for any $s \in \mathbb{R}$

$$
\varphi_{0} \leq \varphi(s) \leq 2 \arccos \left[-r\left(\beta_{x}, \beta_{y}\right)\right]-\varphi_{0}
$$

2. if $\beta_{x} \sin \varphi_{0}+\beta_{y} \cos \varphi_{0}<0$ then, only one of the following holds

a) if $-\pi \leq \varphi_{0}<-\arccos \left[r\left(\beta_{x}, \beta_{y}\right)\right]$ then

$$
\arccos \left[-r\left(\beta_{x}, \beta_{y}\right)\right]-4 \pi-\varphi_{0} \leq \varphi(s) \leq \varphi_{0}
$$

b) if $\arccos \left[-r\left(\beta_{x}, \beta_{y}\right)\right]<\varphi_{0} \leq \pi$ then

$$
2 \arccos \left[-r\left(\beta_{x}, \beta_{y}\right)\right]-\varphi_{0} \leq \varphi(s) \leq \varphi_{0} .
$$

The case $\beta_{x} \sin \varphi_{0}+\beta_{y} \cos \varphi_{0}=0$ is trivial: the solution of (9) is stationary, i.e., $\varphi(s)=\varphi_{0}$ for any $s \in \mathbb{R}$.

The above settles the free end rotation problem.

Remark 2. Let $\varphi(s)$ be the solution to the initial value problem (9).

1. If $\beta_{x} \sin \varphi_{0}+\beta_{y} \cos \varphi_{0}>0$ and $\varphi_{0}>0$, then $\varphi(s)$ has no roots. 
2. If $\beta_{x} \sin \varphi_{0}+\beta_{y} \cos \varphi_{0}>0$ and $\varphi_{0}<0$, then $\varphi(s)$ has one zero because $0 \in\left[\varphi_{0}, 2 \arccos \left[-r\left(\beta_{x}, \beta_{y}\right)\right]-\varphi_{0}\right]$.

3. If $\beta_{x} \sin \varphi_{0}+\beta_{y} \cos \varphi_{0}<0$, then $\varphi(s)$ has no roots, whatever is $\varphi_{0}$.

We can then state:

Theorem 3. If $\varphi_{0}$ is such that

$$
\begin{aligned}
& \beta_{x} \sin \varphi_{0}+\beta_{y} \cos \varphi_{0}>0 \\
& -\frac{\pi}{2}<2 \arctan \left(\frac{\beta_{x}-\sqrt{\beta_{x}^{2}+\beta_{y}^{2}}}{\beta_{y}}\right)<\varphi_{0}<0
\end{aligned}
$$

then, if $L>0$ meets (8), then the solution to (9) will solve (6) also, if and only if $\varphi_{0}$ is a root of

$$
L=\sqrt[4]{\frac{4}{\beta_{x}^{2}+\beta_{y}^{2}}} F\left(\psi\left(\varphi_{0}\right), k\left(\varphi_{0}\right)\right)
$$

with

$$
\begin{aligned}
\psi\left(\varphi_{0}\right) & =\arccos \left(\frac{\beta_{y}+\left(\beta_{x}+\sqrt{\beta_{x}^{2}+\beta_{y}^{2}}\right) \tan \frac{\varphi_{0}}{2}}{\beta_{y}+\left(\beta_{x}-\sqrt{\beta_{x}^{2}+\beta_{y}^{2}}\right) \tan \frac{\varphi_{0}}{2}}\right) \\
k^{2}\left(\varphi_{0}\right) & =\frac{1}{2}+\frac{\beta_{x} \cos \varphi_{0}-\beta_{y} \sin \varphi_{0}}{2 \sqrt{\beta_{x}^{2}+\beta_{y}^{2}}} .
\end{aligned}
$$

Furthermore, the common solution to (9) and (6) is strictly increasing.

Proof: Let us start by noticing that assumptions (12) and (13) ensure the problem to be well posed. Inequality (12) implies that solution (9) is increasing. Using (10) and the boundary condition $\varphi(L)=0$, we get

$$
\int_{\varphi_{0}}^{0} \frac{\mathrm{d} \varphi}{\sqrt{\Phi(\varphi)}}=\int_{\varphi_{0}}^{0} \frac{\mathrm{d} \varphi}{\sqrt{\beta_{x}\left(\sin \varphi-\sin \varphi_{0}\right)-\beta_{y}\left(\cos \varphi-\cos \varphi_{0}\right)}} .
$$

Instead of using formula 293.00 of [4] we will prefer rationalize our integral. If we assume the isoperimetric relationship:

$$
\int_{\varphi_{0}}^{0} \mathrm{~d} s(\varphi)=L
$$


being $L$ the originary rodlength, and put $\varphi=2 \arctan t$, we obtain

$$
L=\frac{2}{\sqrt{-\beta_{x} \sin \varphi_{0}+\beta_{y}\left(1+\cos \varphi_{0}\right)}} \int_{a}^{0} \frac{\mathrm{d} t}{\sqrt{(t-a)(t-b)\left(t^{2}+1\right)}}
$$

where

$$
a=\tan \frac{\varphi_{0}}{2}, \quad b=\frac{\beta_{x}+\beta_{y} \tan \frac{\varphi_{0}}{2}}{\beta_{x} \tan \frac{\varphi_{0}}{2}-\beta_{y}} .
$$

It shall be emphasized that $b<a$ : for the purpose it will be enough to check via elementary calculus that the function

$$
f(\xi)=\frac{\beta_{x}+\beta_{y} \xi}{\beta_{x} \xi-\beta_{y}}-\xi, \quad \frac{\beta_{y}-\sqrt{\beta_{x}^{2}+\beta_{y}^{2}}}{\beta_{x}}<\xi \leq 0
$$

is decreasing and its range is a subset of $\left[-\beta_{x} / \beta_{y}, 0\right)$. Then we can make use of formula 260.00 pag 135 of [4], namely

$$
\int_{a}^{y} \frac{\mathrm{d} t}{\sqrt{(t-a)(t-b)\left(t^{2}+1\right)}}=g F(\psi, k)
$$

where

$$
\begin{gathered}
A^{2}=a^{2}+1, \quad B^{2}=b^{2}+1, \quad g=\frac{1}{\sqrt{A B}} \\
k^{2}=\frac{(A+B)^{2}-(a-b)^{2}}{4 A B}, \quad \psi=\arccos \left[\frac{(A+B) y+a B-b A}{(A+B) y-a B-b A}\right] .
\end{gathered}
$$

The thesis follows after long algebraic work by Mathematica ${ }^{\circledR}$.

Remark 4. Figure 2 confirms that for the $\varphi(s)$ solution to (6), the initial value $\varphi(0)$ is less than zero, as shown in Theorem 3: as a matter of fact the assumed convention on the angle $\theta$ between the oriented tangents will provide certainly $0<\theta_{0}<\pi / 2$, and then $\varphi_{0}<0$.

Corollary 5. If $\varphi_{0}$ is a solution to (14), putting

$$
N(s)=s \sqrt[4]{\frac{\beta_{x}^{2}+\beta_{y}^{2}}{4}}, \quad k^{2}=\frac{1}{2}+\frac{\beta_{y} \cos \varphi_{0}-\beta_{x} \sin \varphi_{0}}{2 \sqrt{\beta_{x}^{2}+\beta_{y}^{2}}}
$$


the solution to (6) will be

$$
\varphi(s)=2 \arctan \frac{p(s)}{q(s)}
$$

where

$$
\begin{aligned}
p(s)= & {\left[\sqrt{\beta_{x}^{2}+\beta_{y}^{2}}\left(1-\cos \varphi_{0} \operatorname{cn}(N(s) \mid k)\right)+\beta_{y} \cos \varphi_{0}(\operatorname{cn}(N(s) \mid k)-1)\right] \beta_{x} } \\
& +\beta_{y}\left(\beta_{y}-\sqrt{\beta_{x}^{2}+\beta_{y}^{2}}\right) \operatorname{cn}(N(s) \mid k) \sin \varphi_{0}+\beta_{x}^{2} \sin \varphi_{0} \\
q(s)= & {\left[\cos \varphi_{0} \operatorname{cn}(N(s) \mid k)+1\right] \beta_{x}^{2}-\beta_{y}\left(\sqrt{\beta_{x}^{2}+\beta_{y}^{2}}-\beta_{y}\right)\left(\cos \varphi_{0}+1\right) } \\
& +\left[(\operatorname{cn}(N(s) \mid k)-1) \beta_{y}+\sqrt{\beta_{x}^{2}+\beta_{y}^{2}}\right] \beta_{x} \sin \varphi_{0} .
\end{aligned}
$$

Proof: It is enough to replace the generic value $\varphi(s)$ to zero at the upper bound of integration in proving Theorem 3.

\section{Elastica Equations $\boldsymbol{x}(s)$ and $\boldsymbol{y}(s)$ Parametrized Via the Arclength}

The formula ruling $\varphi(s)$ introduced in Corollary 5 will be used in order to integrate the differential equations:

$$
x^{\prime}(s)=\cos \varphi(s), \quad y^{\prime}(s)=-\sin \varphi(s) .
$$

For, inserting (16) in (17), we get

$$
x^{\prime}(s)=\frac{q^{2}(s)-p^{2}(s)}{p^{2}(s)+q^{2}(s)}, \quad y^{\prime}(s)=-\frac{2 p(s) q(s)}{p^{2}(s)+q^{2}(s)}
$$

and successively

$$
\begin{aligned}
x^{\prime}(s) & =\frac{a_{0}+a_{1} \mathrm{cn}(N(s) \mid k)+a_{2} \mathrm{cn}^{2}(N(s) \mid k)}{b_{0}+b_{2} \mathrm{cn}^{2}(N(s) \mid k)} \\
y^{\prime}(s) & =\frac{c_{0}+c_{1} \mathrm{cn}(N(s) \mid k)+c_{2} \mathrm{cn}^{2}(N(s) \mid k)}{d_{0}+d_{2} \mathrm{cn}^{2}(N(s) \mid k)}
\end{aligned}
$$


where

$$
\begin{aligned}
& a_{0}=\beta_{x}\left[-8 \beta_{x}^{3} \cos ^{4} \frac{\varphi_{0}}{2}+2 \beta_{x}^{2}\left(4 \sqrt{\beta_{x}^{2}+\beta_{y}^{2}} \cos ^{4} \frac{\varphi_{0}}{2}+\left(2 \sin \varphi_{0}+\sin 2 \varphi_{0}\right) \beta_{y}\right)\right. \\
& +\beta_{x} \beta_{y}\left(\left(-4 \cos \varphi_{0}+\cos 2 \varphi_{0}-3\right) \beta_{y}-2\left(2 \sin \varphi_{0}\right.\right. \\
& \left.\left.\left.+\sin 2 \varphi_{0}\right) \sqrt{\beta_{x}^{2}+\beta_{y}^{2}}\right)+\beta_{y}^{2}\left(4 \beta_{y} \sin \varphi_{0}-\left(\cos 2 \varphi_{0}-3\right) \sqrt{\beta_{x}^{2}+\beta_{y}^{2}}\right)\right] \\
& a_{1}=-4 \beta_{y}\left(\beta_{x} \sin \varphi_{0}+\beta_{y} \cos \varphi_{0}\right)\left[\left(\cos \varphi_{0}+1\right) \beta_{x}^{2}+\left(\sqrt{\beta_{x}^{2}+\beta_{y}^{2}} \sin \varphi_{0}\right.\right. \\
& \left.\left.+\beta_{y}\right) \beta_{y}-\left(\sqrt{\beta_{x}^{2}+\beta_{y}^{2}}\left(\cos \varphi_{0}+1\right)+\beta_{y} \sin \varphi_{0}\right) \beta_{x}\right] \\
& a_{2}=2 \beta_{x}\left(\beta_{x} \sin \varphi_{0}+\beta_{y} \cos \varphi_{0}\right)^{2}\left(\beta_{x}-\sqrt{\beta_{x}^{2}+\beta_{y}^{2}}\right) \\
& b_{0}=2\left[-4 \beta_{x}^{4} \cos ^{4} \frac{\varphi_{0}}{2}+\left(4 \sqrt{\beta_{x}^{2}+\beta_{y}^{2}} \cos ^{4} \frac{\varphi_{0}}{2}+\left(2 \sin \varphi_{0}+\beta_{y} \sin 2 \varphi_{0}\right)\right) \beta_{x}^{3}\right. \\
& -\beta_{x}^{2} \beta_{y}\left(\sqrt{\beta_{x}^{2}+\beta_{y}^{2}}\left(2 \sin \varphi_{0}+\sin 2 \varphi_{0}\right)+\left(2 \cos \varphi_{0}+3\right) \beta_{y}\right) \\
& +\frac{1}{2} \beta_{x} \beta_{y}^{2}\left(\sqrt{\beta_{x}^{2}+\beta_{y}^{2}}\left(4 \cos \varphi_{0}-\cos 2 \varphi_{0}+3\right)+2 \beta_{y}\left(2 \sin \varphi_{0}+\sin 2 \varphi_{0}\right)\right) \\
& \left.-\beta_{y}^{3}\left(2 \sqrt{\beta_{x}^{2}+\beta_{y}^{2}} \sin \varphi_{0}+\left(\sin ^{2} \varphi_{0}+1\right) \beta_{y}\right)\right] \\
& b_{2}=-2\left(\beta_{x} \sin \varphi_{0}+\beta_{y} \cos \varphi_{0}\right)^{2}\left(\beta_{x}^{2}+\beta_{y}^{2}-\beta_{x} \sqrt{\beta_{x}^{2}+\beta_{y}^{2}}\right) \\
& c_{0}=\beta_{y}\left(-\beta_{x} \cos \varphi_{0}+\beta_{y} \sin \varphi_{0}+\sqrt{\beta_{x}^{2}+\beta_{y}^{2}}\right)\left[\left(-\cos \varphi_{0}-1\right) \beta_{x}^{2}\right. \\
& \left.+\left(\left(\cos \varphi_{0}+1\right) \sqrt{\beta_{x}^{2}+\beta_{y}^{2}}+\beta_{y} \sin \varphi_{0}\right) \beta_{x}-\beta_{y}\left(\sqrt{\beta_{x}^{2}+\beta_{y}^{2}} \sin \varphi_{0}+\beta_{y}\right)\right] \\
& c_{1}=-2 \beta_{x}\left(\beta_{x} \sin \varphi_{0}+\beta_{y} \cos \varphi_{0}\right)\left[\left(\cos \varphi_{0}+1\right) \beta_{x}^{2}+\beta_{y}\left(\sqrt{\beta_{x}^{2}+\beta_{y}^{2}} \sin \varphi_{0}\right.\right. \\
& \left.\left.+\beta_{y}\right)-\left(\left(\cos \varphi_{0}+1\right) \sqrt{\beta_{x}^{2}+\beta_{y}^{2}}+\beta_{y} \sin \varphi_{0}\right) \beta_{x}\right] \\
& c_{2}=\beta_{y}\left(\beta_{x} \sin \varphi_{0}+\beta_{y} \cos \varphi_{0}\right)^{2}\left(\sqrt{\beta_{x}^{2}+\beta_{y}^{2}}-\beta_{x}\right) \\
& d_{0}=4 \beta_{x}^{4} \cos ^{4} \frac{\varphi_{0}}{2}-\left(4 \sqrt{\beta_{x}^{2}+\beta_{y}^{2}} \cos ^{4} \frac{\varphi_{0}}{2}+\left(2 \sin \varphi_{0}+\sin 2 \varphi_{0}\right) \beta_{y}\right) \beta_{x}^{3} \\
& +\beta_{x}^{2} \beta_{y}\left(\sqrt{\beta_{x}^{2}+\beta_{y}^{2}}\left(2 \sin \varphi_{0}+\sin 2 \varphi_{0}\right)+\left(2 \cos \varphi_{0}+3\right) \beta_{y}\right) \\
& +\beta_{y}^{3}\left(2 \sqrt{\beta_{x}^{2}+\beta_{y}^{2}} \sin \varphi_{0}+\left(\sin ^{2} \varphi_{0}+1\right) \beta_{y}\right) \\
& -\frac{1}{2} \beta_{x} \beta_{y}^{2}\left(\sqrt{\beta_{x}^{2}+\beta_{y}^{2}}\left(4 \cos \varphi_{0}-\cos 2 \varphi_{0}+3\right)+2 \beta_{y}\left(2 \sin \varphi_{0}+\sin 2 \varphi_{0}\right)\right) \\
& d_{2}=\left(\beta_{x} \sin \varphi_{0}+\beta_{y} \cos \varphi_{0}\right)^{2}\left(\beta_{x}^{2}+\beta_{y}^{2}-\beta_{x} \sqrt{\beta_{x}^{2}+\beta_{y}^{2}}\right) .
\end{aligned}
$$


Notice that $b_{0}$ and $b_{2}$ are negative, while $d_{0}$ and $d_{2}$ are positive, and

$$
\frac{d_{0}}{b_{0}}=\frac{d_{2}}{b_{2}}=-\frac{1}{2} \text {. }
$$

Moreover, the following fundamental relations hold

$$
k^{2}=\frac{b_{2}}{b_{0}+b_{2}}=\frac{d_{2}}{d_{0}+d_{2}} .
$$

After this preliminaries, we can now integrate (18) and (19).

Theorem 6. The elastica parametric equations are

$$
x(s)=\frac{a_{2}}{b_{2}} s+\frac{\sqrt{2}}{\left(b_{0}+b_{2}\right) \sqrt[4]{\beta_{x}^{2}+\beta_{y}^{2}}}\left[\frac{a_{0} b_{2}-a_{2} b_{0}}{b_{2}} \Pi\left(k^{2}, k, N(s)\right)+a_{1} \operatorname{sd} N(s)\right]
$$

$y(s)=\frac{c_{2}}{d_{2}} s+\frac{\sqrt{2}}{\left(d_{0}+d_{2}\right) \sqrt[4]{\beta_{x}^{2}+\beta_{y}^{2}}}\left[\frac{c_{0} d_{2}-c_{2} d_{0}}{d_{2}} \Pi\left(k^{2}, k, N(s)\right)+c_{1} \operatorname{sd} N(s)\right]$

where $\Pi$ is the third kind incomplete elliptic integral and, following Glaisher, we mean

$$
\operatorname{sd} x=\frac{\operatorname{sn} x}{\operatorname{dn} x} .
$$

Proof: Both integrations in (21) are of the same nature, and we will consider only the first one. Splitting in partial fractions, and omitting the modulus $k$, by identities

$$
\begin{aligned}
& \frac{a_{0}+a_{1} z+a_{2} z^{2}}{b_{0}+b_{2} z^{2}}=\frac{a_{2}}{b_{2}}+\frac{\frac{a_{0} b_{2}-a_{2} b_{0}}{b_{2}}+a_{1} z}{b_{0}+b_{2} z^{2}} \\
& b_{0}+b_{2} \operatorname{cn}^{2} \sigma=\left(b_{0}+b_{2}\right)\left(1-\frac{b_{2}}{b_{0}+b_{2}} \operatorname{sn}^{2} \sigma\right)
\end{aligned}
$$

one finds

$$
x(s)=\frac{a_{2}}{b_{2}} s+\frac{\sqrt{2}}{\left(b_{0}+b_{2}\right) \sqrt[4]{\beta_{x}^{2}+\beta_{y}^{2}}}\left[\frac{a_{0} b_{2}-a_{2} b_{0}}{b_{2}} I_{x 1}(s)+a_{1} I_{x 2}(s)\right]
$$

with

$$
I_{x 1}(s)=\int_{0}^{N(s)} \frac{\mathrm{d} \sigma}{1-k^{2} \mathrm{sn}^{2} \sigma}, \quad I_{x 2}(s)=\int_{0}^{N(s)} \frac{\operatorname{cn} \sigma \mathrm{d} \sigma}{1-k^{2} \operatorname{sn}^{2} \sigma} .
$$


The first integral is given by [4] formula 336.01: $I_{x 1}(s)=\Pi\left(k^{2}, k, N(s)\right)$. In order to compute $I_{x 2}(s)$, putting $\operatorname{sn}^{2} \sigma=w$, we get: $2 \operatorname{sn} \sigma \operatorname{cn} \sigma \operatorname{dn} \sigma \mathrm{d} \sigma=\mathrm{d} w$, where $\operatorname{dn} \sigma$ is the Jacobi delta amplitude elliptic function $\operatorname{dn}^{2} \sigma=1-k^{2} \operatorname{sn}^{2} \sigma$. We find out

$$
I_{x 2}(s)=\frac{1}{2} \int_{0}^{\mathrm{sn}^{2} N(s)} \frac{\mathrm{d} w}{\left(1-k^{2} w\right) \sqrt{w\left(1-k^{2} w\right)}} .
$$

Putting $z=\frac{1}{k} \sqrt{\frac{1-k^{2} w}{w}}$, we get $I_{x 2}(s)=\operatorname{sd} N(s)$.

\section{A Generalization}

The next step could be to establish what happens if a bending couple $\mathfrak{M}_{0}$ is added as a further load to the free end. One could think that our solution can cover the free end simultaneously loaded by a force and a couple $\mathfrak{M}_{0}$. Of course the couple is not whichever, but its plane shall be parallel to that of rod and force. In such a case (3) shall be replaced by

$$
M_{z}(s) \pm \mathfrak{M}_{0}=-E J \frac{\mathrm{d} \varphi(s)}{\mathrm{d} s}
$$

whilst ODE (5) in $\varphi(s)$ is affected only at the free end boundary condition, which becomes:

$$
\frac{\mathrm{d} \varphi}{\mathrm{d} s}(0)=\frac{\mp \mathfrak{M}_{0}}{E J} .
$$

Then we will find a different $\varphi(s)$. The $\varphi_{0}$ evaluation is not involved by the $\mathfrak{M}_{0}$ addition. On the contrary, the $x$ and $y$ functional expressions will be conditioned by the new $\varphi(s)$ only.

\section{Conclusions}

A thin, flexible cantilever is studied in its elastic equilibrium configuration under a concentrated terminal load. In order to make the problem tractable, a proper set of curvilinear state variables is chosen, facing with a second order, nonlinear, but autonomous ODE to be solved to rotation $\varphi(s)$ pertaining to each $s$-section. This planar problem, even if relevant to linear elasticity, is a nonlinear one; and even if ruled by a second order ODE it is not a Cauchy problem. Nevertheless its solution depends in some extent on auxiliary Cauchy problem. The evaluation of the free 
end rotation $\varphi_{0}$, following the isoperimetric assumption, leads to a numerical subproblem inside the main BVP, which is solved via the shooting approach starting from an auxiliary, suitably chosen IVP. Furthermore, if $x(s)$ and $y(s)$ denote the elastica coordinates parametrized by the arclength $s$, through a first integration one obtains $x^{\prime}(s)$ and $y^{\prime}(s)$ as elliptic functions of $s$. Further, some minor changes are shown in order to pass from a loading force to a more general free-end load combination of a force and a couple. Anyway, performing the second integration, the elastica parametric equations are obtained in closed form. It has been found that $x$ and $y$ are consisting of the addition of three functional blocks:

1. the first is proportional to the arc itself

2. the second is proportional to the third kind elliptic integral, whose upper bound is the arc itself

3. the third is the elliptic function sd of the arc.

The functional links are therefore quite tractable and on the contrary, the constants involved have a so long formulation in terms of problem data, that we preferred to provide for them directly the relevant output of our Mathematica ${ }^{\circledR}$ computer algebra package. Finally, the reader should be aware that all our symbolic formulations have been tested affronting some sample problems and comparing the outputs coming from our closed form solutions with the numerical ones from high-reliability Runge-Kutta schemes.

\section{Acknowledgments}

The authors thank very warmly their friend Aldo Scimone who drew all figures of this paper.

\section{References}

[1] Agostinelli C. and Pignedoli A., Meccanica Razionale, vol. 1, Zanichelli, Bologna, 1978.

[2] Bailey P., Shampine L. and Waltman P. Nonlinear Two Point Boundary Value Problems, Academic Press, New York, 1974.

[3] Bisshopp K. and Drucker D., Large Deflection Cantilever Beams, Quart. Appl. Math. 3 (1945) 272-275. 
[4] Byrd P. and Friedman M., Handbook of Elliptic Integrals for Engineers and Scientists, Springer, New York, 1971.

[5] Howell L. and Midha A., Parametric Deflection Approximations for Endloaded Large Deflection Beams in Compliant Mechanisms, ASME J. Mech. Des. 117 (1995) 156-165.

[6] Kimball C. and Tsai L.-W., Modeling of Flexural Beams Subjected to Arbitrary End Loads, ASME J. Mech. Des. 124 (2002) 223-234.

[7] Landau L. and Lifchitz E., Théorie de l'élasticité, MIR, Moscow 1967.

[8] Love A. E. H., A Treatise on the Mathematical Theory of Elasticity, Dover, New York, 1944.

[9] Scarpello G. and Ritelli D., Elliptic Integrals Solution to Elastica's Boundary Value Problem of a Rod Bent by Axial Compression, J. Anal. Appl. 5 (2007) 53-69.

[10] Neukirch S. and Henderson M., Classification of the Spatial Clamped Elastica: Symmetries and Zoology of Solutions, J. Elasticity 68 (2002) 95-121.

[11] Nizette M. and Goriely A., Toward a Classification of Euler-Kirchhoff Filaments, J. Math. Phys. 40 (1999) 2830-2866.

Giovanni Mingari Scarpello

Dipartimento di Matematica

per le Scienze Economiche e Sociali

viale Filopanti, 540126 Bologna

ITALY

E-mail address: giovannimingari@yahoo.it

Daniele Ritelli

Dipartimento di Matematica

per le Scienze Economiche e Sociali

viale Filopanti, 540126 Bologna

ITALY

E-mail address: daniele.ritelli@unibo.it 